
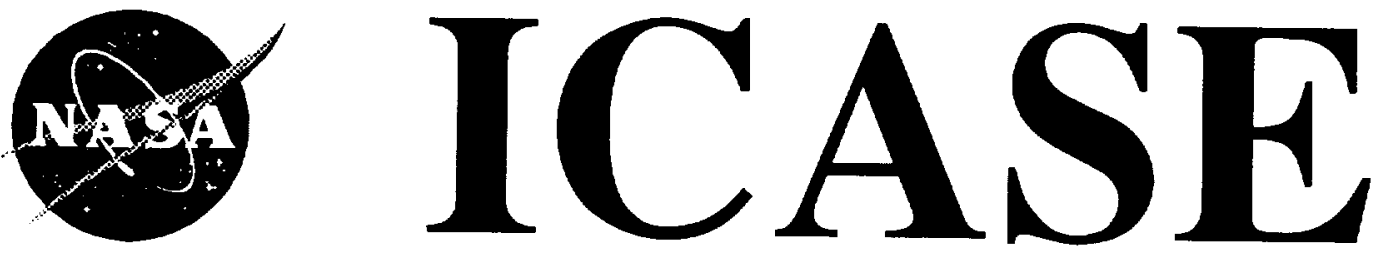

\title{
THE EFFECTS OF VISCOSITY ON THE STABILITY OF A TRAILING-LINE VORTEX IN COMPRESSIBLE FLOW
}

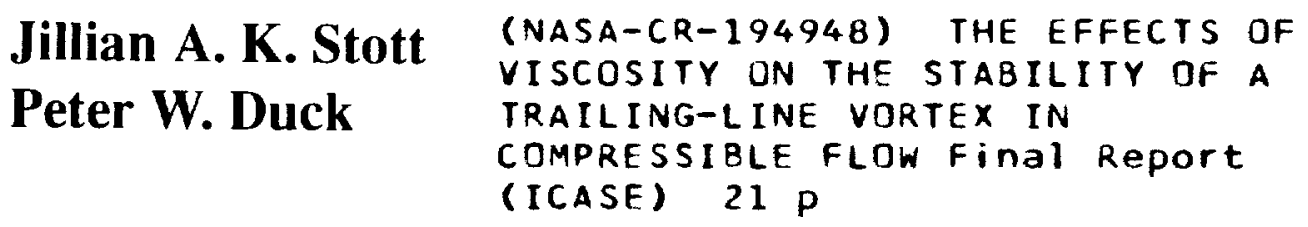

N95-11169

Unclas

$63 / 34 \quad 0019857$

Contract NAS1-19480

July 1994

Institute for Computer Applications in Science and Engineering NASA Langley Research Center

Hampton, VA 23681-0001

SRA Operated by Universities Space Research Association 


\section{ICASE Fluid Mechanics}

Due to increasing research being conducted at ICASE in the field of fluid mechanics, future ICASE reports in this area of research will be printed with a green cover. Applied and numerical mathematics reports will have the familiar blue cover, while computer science reports will have yellow covers. In all other aspects the reports will remain the same; in particular, they will continue to be submitted to the appropriate journals or conferences for formal publication. 


\title{
The effects of viscosity on the stability of a trailing-line vortex in compressible flow
}

\author{
by \\ Jillian A. K. Stott \\ and \\ Peter W. Duck* \\ Department of Mathematics, \\ University of Manchester, \\ Manchester, M13 9PL, \\ United Kingdom
}

\begin{abstract}
We consider the effects of viscosity on the inviscid stability of the Batchelor (1964) vortex in a compressible flow. The problem is tackled asymptotically, in the limit of large (streamwise and azimuthal) wavenumbers, together with large Mach numbers. This problem, with viscous effects neglected, was discussed in Stott \& Duck (1994). The authors found that the nature of the solution passes through different regimes as the Mach number increases, relative to the wavenumber. This structure persists when viscous effects are included in the analysis. In the present study, as in that mentioned above, the mode present in the incompressible case ceases to be unstable at high Mach numbers and a centre mode forms, whose stability characteristics are determined primarily by conditions close to the vortex axis.

We find generally that viscosity has a stabilising influence on the flow, whilst in the case of centre modes, viscous effects become important at much larger Reynolds numbers than for the first class of disturbance.
\end{abstract}

* This work was partially supported by the National Aeronautics and Space Administration under NASA Contract No. NAS1-19480 while the author was in residence at the Institute for Computer Applications in Science and Engineering (ICASE), NASA Langley Research Center, Hampton, VA, 23681-0001. 


$+4+\infty$




\section{Introduction and formulation}

The work presented in this paper is a follow on study to that of Stott \& Duck (1994), where the inviscid stability of the Batchelor (1964) trailing-line vortex in a compressible flow was considered. The motivation for this research is due to relevance to tornadoes, combustion optimisation and also the breakdown of such vortices behind aircraft.

The first authors to investigate viscous effects in the study of the stability of the Batchelor (1964) vortex were Lessen \& Paillet (1974). A numerical approach was adopted and it was shown the $n=-1$ mode (where $n$ is the azimuthal wavenumber) was the most unstable mode. This finding was contrary to previous inviscid studies i.e. Lessen, Singh \& Paillet (1974), which had indicated that disturbances with $-n \gg 1$ were the most dangerous. However the work of Lessen, Singh \& Paillet (1974) considered only relatively low Reynolds numbers (i.e. $R e \sim 100$ ), which is unrealistic for comparison with experimental flow situations and with inviscid studies. This apparent anomaly remained unresolved until the study by Stewartson (1982), in which the problem was approached asymptotically (based on the inviscid work of Leibovich \& Stewartson 1983) which considered the dual limits of large Reynolds numbers and wavenumbers. The results obtained show that the effects of viscosity are stabilising and also the increase in the growth rates as $-n$ increases is not sufficient to counter the stabilising effects of viscosity which may be quite significant at low Reynolds numbers.

In the past few years it has also been established that additional unstable modes exist in which viscosity exerts a destabilising influence. These viscous modes were first discussed by Khorrami (1991) and later analysed by Duck \& Khorrami (1992). Also Khorrami (1992) looked at the full viscous incompressible equations by numerical techniques and found that at large Reynolds numbers, with $n=-1$, near the upper neutral point, the first and second modes cross and switch. In addition a second peak in the growth rate curves appears due to viscous effects.

In this paper, we take cylindrical polar coordinates, $(L \hat{r}, \theta, L x)$, with the $x$-axis lying along the axis of the vortex (which is taken to be axisymmetric), and $L$ is some streamwise scale. We also take the flow far from the vortex centre to be directed along the $x$-direction. The velocity field is written as $U_{s}^{*} \mathbf{u}=U_{s}^{*}(u, v, w)$, the fluid density is $\rho_{\infty}^{*} \rho$, temperature $T_{\infty}^{*} T$, first and second coefficients of viscosity $\mu_{\infty}^{*} \mu, \mu_{\infty}^{*} \lambda$ respectively, and pressure $\rho_{\infty}^{*} U_{s}^{* 2} p$. Here superscript asterisk and subscript $\infty$ respectively denote dimensional and freestream variables. $U_{s}^{*}$ is a velocity scale, defined by

$$
U_{s}^{*}=\frac{C_{0} U_{\infty}^{*}}{8 x \nu_{\infty}^{*}{ }^{2} R e_{\infty}} \log \left(x R e_{\infty}\right)+\frac{L U_{\infty}^{*}{ }^{2}}{8 x \nu_{\infty}^{*}{ }^{2} R e_{\infty}}
$$

where $U_{\infty}^{*}$ is the freestream velocity, $\nu_{\infty}=\mu_{\infty}^{*} / \rho_{\infty}^{*}$ is the kinematic viscosity, $C_{0}$ is a constant and $R e_{\infty}$ is the freestream Reynolds number defined by

$$
R e_{\infty}=\frac{\rho_{\infty}^{*} U_{\infty}^{*} L}{\mu_{\infty}^{*}}
$$

We have a flow Mach number given by

$$
M=\frac{U_{s}^{*}}{\left(\gamma R^{*} T_{\infty}^{*}\right)^{\frac{1}{2}}}
$$


where $\gamma$ is the ratio of specific heats and $R^{*}$ the gas constant. The freestream Mach number is given by

$$
M_{\infty}=\frac{U_{\infty}^{*}}{U_{s}^{*}} M,
$$

where we expect $M_{\infty} \gg M$. Also we define the Prandtl number to be

$$
\sigma=\frac{\mu^{*} c_{p}}{\kappa^{*}}
$$

where $\kappa^{*}$ is the thermal conductivity of the fluid and $c_{p}$ is the specific heat at constant pressure. We further assume a perfect fluid.

The flow of a swirling wake in the far downstream limit was first discussed by Batchelor (1964) where the flow was taken to be incompressible and a similarity solution was sought. This work was extended into the compressible regime by Stott \& Duck (1994) who showed the following non-dimensional profile was appropriate to leading order

$$
\begin{array}{lrl}
U=e^{-r^{2}}, & p_{0}=\frac{U_{\infty}^{2}}{U_{s}^{2}}, & V=0, \\
T_{0}=1, & W=\frac{q}{r}\left(1-e^{-r^{2}}\right), & \rho_{0}=1,
\end{array}
$$

where $q$ is a measure of swirl, and

$$
r=\frac{\hat{r}}{r_{s}}=\frac{\hat{r} R e_{\infty}^{\frac{1}{2}}}{4 x}
$$

It is (6) that we shall be adopting as the basic flow profile for the stability study considered in the main part of this paper. We also note that the flow Reynolds number is defined by

$$
R e=\frac{\rho_{\infty}^{*} U_{s}^{*} r_{s}}{\mu_{\infty}^{*}}
$$

Consider now the stability equations of the general basic state; taking small perturbations, we write

$$
\begin{gathered}
(u, v, w, p, T, \rho)=\left(U(r), 0, W(r), p_{0}, T_{0}\right) \\
+\delta\left[F(r), i G(r), H(r), \frac{P(r)}{n}, \tau(r), \Gamma(r)\right] e^{i\left(\alpha(x-c t) / r_{\varepsilon}+n \theta\right)}+\mathrm{O}\left(\delta^{2}\right)
\end{gathered}
$$

where $\delta \ll 1$ and $c=c_{r}+c_{i}$ is the complex wavespeed. We assume that $\mu^{*}$, the first coefficient of viscosity, is solely a function of the temperature $T^{*}$. In addition we also take 
the bulk viscosity to be zero. If we now substitute equations (9) and the above assumptions into the full Navier-Stokes equations, and consider only terms of $O(\delta)$, we obtain

$$
\begin{aligned}
& \varphi F+U^{\prime} G+\frac{\alpha P}{n}=\frac{1}{i R e}\left[\frac{1}{r}\left(r F^{\prime}\right)^{\prime}-\left(\alpha^{2}+\frac{n^{2}}{r^{2}}\right) F+\alpha \Gamma \varphi+\frac{1}{r}\left(r \tau \frac{d \mu}{d T_{0}} U^{\prime}\right)^{\prime}\right], \\
& \varphi G+\frac{2 W H}{r}+\frac{\Gamma W^{2}}{r}=\frac{P^{\prime}}{n}+\frac{1}{i R e}\left[\frac{1}{r}\left(r G^{\prime}\right)^{\prime}-\left(\alpha^{2}+\frac{n^{2}+1}{r^{2}}\right) G\right. \\
& \left.-(\Gamma \varphi)^{\prime}-\frac{2 n H}{r^{2}}+n \tau \frac{d \mu}{d T_{0}}\left(\frac{W}{r}\right)^{\prime}+\alpha \tau \frac{d \mu}{d T_{0}} U^{\prime}\right] \\
& \varphi H+\bar{W}^{+} G+\frac{P}{r}=\frac{1}{i \operatorname{Re}}\left[\frac{1}{r}\left(r H^{\prime}\right)^{\prime}-\left(\alpha^{2}+\frac{n^{2}+1}{r^{2}}\right) H-\frac{2 n G}{r^{2}}\right. \\
& \left.+\left(r \tau \frac{d \mu}{d T_{0}}\left(\frac{W}{r}\right)^{\prime}\right)^{\prime}+2 \tau \frac{d \mu}{d T_{0}}\left(\frac{W}{r}\right)^{\prime}+n \frac{\Gamma \varphi}{r}\right] \\
& \Gamma \varphi+\alpha F+\frac{n H}{r}=-G^{\prime}-\frac{G}{r} \\
& \varphi \tau-\frac{(\gamma-1) M^{2} \varphi P}{n}=\frac{1}{\sigma i R e}\left[\frac{1}{r}\left(r \tau^{\prime}\right)^{\prime}-\left(\alpha^{2}+\frac{n^{2}}{r^{2}}\right) \tau\right] \\
& +\frac{M^{2}(\gamma-1)}{i R e}\left[-\frac{2 n}{r} \bar{W} G+2 \bar{W}\left(H^{\prime}-\frac{H}{r}\right)+\tau \frac{d \mu}{d T_{0}} \bar{W}^{2}+2 U^{\prime}\left(F^{\prime}-\alpha G\right)+\tau \frac{d \mu}{d T_{0}} U^{\prime 2}\right], \\
& \frac{\gamma M^{2} P}{n}=\Gamma+\tau
\end{aligned}
$$

where

$$
\overline{W^{+}}=\left(W^{\prime}+\frac{W}{r}\right), \quad \bar{W}=\left(W^{\prime}-\frac{W}{r}\right), \quad \varphi=\alpha(U-c)+\frac{n W}{r} .
$$

Also note that for this study we choose the Prandtl number to be unity and for the purposes of the results presented later the ratio of specific heats is taken to be 1.4, although of course there is no conceptual difficulty in choosing other values.

The boundary conditions are given by

$$
\begin{array}{lll}
F^{\prime}(0)=G(0)=H(0)=P^{\prime}(0)=\Gamma^{\prime}(0)=\tau^{\prime}(0)=0 & \text { for } & n=0 \\
F(0)=G(0)+H(0)=P(0)=\Gamma(0)=\tau(0)=0 & \text { for } & |n|=1 \\
F(0)=G(0)=H(0)=P(0)=\Gamma(0)=\tau(0)=0 & \text { for } & |n|>1
\end{array}
$$




$$
F, G, H, P, \Gamma, \tau \rightarrow 0 \quad \text { as } \quad r \rightarrow \infty .
$$

Unlike the inviscid analysis of Stott \& Duck (1994), we are unable to reduce the equations further, and thus (in the temporal problem), (10) defines the eigenvalue problem for $c$.

In section 2 we develop an asymptotic theory for large wavenumbers when viscous effects are included in the analysis. In section 3 it is shown that for sufficiently large Mach numbers centre modes are found and consideration is made of the effect of viscosity on these modes; indeed, the authors are unaware of previous analysis of the effects of viscosity on any kind of centre modes. In section 4 we present and discuss results obtained from the analysis of sections 2 and 3 .

\section{Analysis for large azimuthal wavenumbers. $\mathbf{M}=\mathbf{O}\left(|\mathbf{n}|^{\frac{1}{2}}\right)$}

It was shown in the inviscid work of Stott \& Duck (1994) that the crucial regime of Mach number is when $M=O\left(|n|^{\frac{1}{2}}\right)$. In this case there is an important change in the character of the inviscid instability. The same is expected to be true in the viscous case, if conditions are appropriately chosen. The $M=O\left(|n|^{\frac{1}{2}}\right)$ regime results also encompass, to a large extent, many of the results of smaller orders of $M$. We then write $M=|n|^{\frac{1}{2}} \widehat{M}, \widehat{M}=O(1)$, together with $\alpha=n \bar{\alpha}$, and the wavespeed is then expected to develop in the form

$$
c=c_{0}+\frac{c_{1}}{n}+\frac{c_{2}}{|n|^{\frac{3}{2}}}+\mathrm{O}\left(\frac{1}{n^{2}}\right) .
$$

It is found that the crucial order of the Reynolds number (defined in (8)) is given by $R e=\frac{-i n^{2}}{\hat{\mu}}$ where $\hat{\mu}=O(1)$ is the (imaginary) viscosity parameter. Although viscosity is a function of temperature, since the temperature is (approximately) constant, then so too is $\hat{\mu}$.

Following the inviscid analysis of Stott \& Duck (1994), we fully expect the eigensolutions to be concentrated close to the point where $\varphi_{0}\left(r_{0}\right)=0$, together with $\varphi_{0}^{\prime}\left(r_{0}\right)=0$, where

$$
\varphi_{0}(r)=\bar{\alpha}\left(U-c_{0}\right)+\frac{W}{r} .
$$

The relevant radial scale is then

$$
R=\left(r-r_{0}\right)|n|^{\frac{3}{4}} \text {. }
$$

On the $R=O(1)$ scale, $\varphi$ develops in the form

$$
\varphi=\varphi_{1}+\frac{\varphi_{2}}{|n|^{\frac{1}{2}}}+\mathrm{O}\left(\frac{1}{|n|}\right) .
$$

We then expect $F, G, H, P, \tau, \Gamma$ to take the form

$$
\begin{gathered}
(F, G, H, P, \tau, \Gamma)=\left(F_{0}, G_{0}, H_{0}, P_{0}, \tau_{0}, \Gamma_{0}\right)+\frac{1}{|n|^{\frac{1}{4}}}\left(F_{1}, G_{1}, H_{1}, P_{1}, \tau_{1}, \Gamma_{1}\right) \\
+\frac{1}{|n|^{\frac{1}{2}}}\left(F_{2}, G_{2}, H_{2}, P_{2}, \tau_{2}, \Gamma_{2}\right)+\ldots
\end{gathered}
$$


Taking the $O(1)$ terms in the governing equations (10) leads to

$$
\begin{aligned}
& G_{0}=-\frac{\widehat{\varphi}_{1}\left(1+\bar{\alpha}^{2} r_{0}^{2}\right)}{U_{0}^{\prime}-\bar{\alpha} r_{0} \bar{W}_{0}^{+}} F_{0}, \\
& H_{0}=-\bar{\alpha} r_{0} F_{0}, \\
& P_{0}=\frac{2 W_{0} \widehat{\varphi}_{1}}{U_{0}^{\prime}-\bar{\alpha} r_{0} \overline{W_{0}^{+}}} F_{0}, \\
& \Gamma_{0}=\frac{r_{0}}{W_{0}^{2}}\left(\frac{\widehat{\varphi}_{1}^{2}\left(1+\bar{\alpha}^{2} r_{0}^{2}\right)}{U_{0}^{\prime}-\bar{\alpha} r_{0} \bar{W}_{0}^{+}}+2 \bar{\alpha} W_{0}\right) F_{0},
\end{aligned}
$$

where $\widehat{\varphi}_{1}=\varphi_{1}+\frac{\hat{\mu}}{r_{0}^{2}}\left(1+\bar{\alpha}^{2} r_{0}^{2}\right)$, and then $\widehat{\varphi}_{1}$ is given by the solution of the following quadratic equation

$$
\begin{gathered}
\widehat{\varphi}_{1}^{2}\left(1+\bar{\alpha}^{2} r_{0}^{2}\right)-\frac{2 \operatorname{sign}(n) \widehat{M}^{2} W_{0}^{3}}{r_{0}} \widehat{\varphi}_{1} \\
-\left(2 \operatorname{sign}(n) W_{0}^{3} \widehat{M}^{2} \frac{(\gamma-1) \hat{\mu}\left(1+\bar{\alpha}^{2} r_{0}^{2}\right)}{r_{0}^{3}}-2 \bar{\alpha} W_{0}\left(U_{0}^{\prime}-\bar{\alpha} r_{0} \overline{W_{0}}\right)\right)=0
\end{gathered}
$$

and hence from this we may deduce $c_{1}$ (since $\varphi_{1}=-\bar{\alpha} c_{1}$ ). Continuing, by equating terms of $\mathrm{O}\left(|n|^{-\frac{1}{4}}\right)$, we have that

$$
\begin{aligned}
& F_{1}=\operatorname{sign}(n)\left(\frac{\bar{\alpha} r_{0}^{2} \widehat{\varphi}_{1}}{U_{0}^{\prime}-\bar{\alpha} r_{0} \overline{W_{0}^{+}}}\right) \frac{d F_{0}}{d R}+A_{1} F_{0}, \\
& G_{1}=-\frac{A_{1} \widehat{\varphi}_{1}\left(1+\bar{\alpha}^{2} r_{0}^{2}\right)}{U_{0}^{\prime}-\bar{\alpha} r_{0} \overline{W_{0}^{+}}} F_{0}, \\
& H_{1}=\operatorname{sign}(n)\left(\frac{r_{0} \widehat{\varphi}_{1}}{U_{0}^{\prime}-\bar{\alpha} r_{0} \overline{W_{0}^{+}}}\right) \frac{d F_{0}}{d R}-\bar{\alpha} r_{0} A_{1} F_{0}, \\
& P_{1}=\operatorname{sign}(n)\left(\frac{-r_{0}^{2} \widehat{\varphi}_{1}^{2}}{U_{0}^{\prime}-\bar{\alpha} r_{0} \overline{W_{0}^{+}}}\right) \frac{d F_{0}}{d R}+2 \bar{\alpha} W_{0} A_{1} F_{0}, \\
& \Gamma_{1}=\frac{A_{1} r_{0}}{W_{0}^{2}}\left(\frac{\widehat{\varphi}_{1}^{2}\left(1+\bar{\alpha}^{2} r_{0}^{2}\right)}{U_{0}^{\prime}-\bar{\alpha} r_{0} \bar{W}_{0}^{+}}+2 \bar{\alpha} W_{0}\right) F_{0},
\end{aligned}
$$

where $A_{1}$ is an undetermined constant. On account of (20), a solution only exists if the following equation is satisfied 


$$
F_{0 R R}-\left(\hat{\lambda}_{1} R^{2}+\hat{\lambda}_{0} c_{2}\right) F_{0}=0
$$

where

$$
\begin{aligned}
& \hat{\lambda}_{0}=-\frac{\bar{\alpha}\left(2 \operatorname{sign}(n) \widehat{M}^{2} W_{0}^{3} \widehat{\varphi}_{1}+2 \widehat{\varphi}_{1}^{2}\left(1+\bar{\alpha}^{2} r_{0}^{2}\right) r_{0}\right)}{\operatorname{sign}(n) 2 W_{0}^{3} \widehat{\varphi}_{1} \gamma \widehat{M}^{2} \hat{\mu}-2 \hat{\varphi}_{1}^{2}\left(1+\bar{\alpha}^{2} r_{0}^{2}\right) \hat{\mu}-\hat{\varphi}_{1}^{3} r_{0}^{2}}, \\
& \hat{\lambda}_{1}=\frac{-\varphi_{0}^{\prime \prime}\left(r_{0}\right)}{2 \bar{\alpha}} .
\end{aligned}
$$

Equation (22) is of the form of Weber's equation (after the application of the transformation $\xi=2^{\frac{1}{2}} \hat{\lambda}_{1}^{\frac{1}{4}} R$ ). Therefore, in order to satisfy the boundary conditions we must have solutions of the form

$$
c_{2}=-\frac{\hat{\lambda}_{1}^{\frac{1}{2}}(1+2 m)}{\hat{\lambda}_{0}} \quad m=0,1,2 \ldots
$$

We note that setting $\hat{\mu}$, the viscosity parameter, to zero reduces the problem to its inviscid counterpart as considered by Stott \& Duck (1994). Also we see that when $|\hat{\mu}|$ becomes sufficiently large, all disturbances become neutrally stable.

As in the case of inviscid flow, this regime of $M$ indicates a watershed in our analysis. When $\widehat{M}$ is not large, both roots of $c_{1}$ (obtained from equation (20)) are complex and hence the flow is unstable. However, increasing $\widehat{M}$ eventually causes $c_{1}$ to become purely real, which implies $c_{2}$ also becomes real. Consequently to this order of approximation, for sufficiently large $\widehat{M}$, all disturbances become neutrally stable. The effects of viscosity on this phenomenon and other results obtained from this section will be discussed again in section 4 .

We expect, as in the inviscid case, the emergence of centre modes as $\widehat{M}$ becomes large, and it is this class of modes that comes under consideration in the next section.

\section{Centre Modes. $\mathbf{M}=\mathbf{O}(|\mathbf{n}|)$}

We anticipate in this regime, as in the inviscid case, that centre modes (i.e. modes whose disturbances are primarily dictated by conditions close to the axis of the flow) will exist. We set $M=|n| \bar{M}, \quad \bar{M}=\mathrm{O}(1)$ and assume that disturbances will develop in a similar way to those found by Stott \& Duck (1994). Thus we expect the flow to be divided into five regions, two regions within $r=\mathrm{O}(1)$ (one above, the other below a critical layer), $r=\mathrm{O}\left(|n|^{-\frac{1}{2}}\right)$ and finally $r=\mathrm{O}\left(|n|^{-1}\right)$. Also, as in the purely inviscid case, we expect the complex wavespeed to develop as

$$
c=c_{0}+\frac{c_{1}}{n^{2}}+\ldots
$$


and therefore

$$
\begin{aligned}
\varphi & =n \varphi_{0}+\frac{\varphi_{1}}{n}+\ldots \\
& =n\left\{\bar{\alpha}\left(U-c_{0}\right)+\frac{W}{r}\right\}+\left\{-\frac{\bar{\alpha} c_{1}}{n}+\frac{n}{2} \varphi_{0}^{\prime \prime}(0) r^{2}\right\}+\ldots .
\end{aligned}
$$

For self-consistency, it turns out that we must have $\varphi_{0}(r=0)=0$, i.e.

$$
c_{0}=1+\frac{q}{\bar{\alpha}}
$$

We first consider the inner lengthscale, $R=r|n|=\mathrm{O}(1)$. The other regimes, mentioned earlier, will be discussed briefly later, since details are identical to the inviscid case.

The solution is somewhat complicated by the fact that (as seen in the inviscid solution of Stott \& Duck 1994) the solution may be regarded as of WKB/multiple scales type. In the case of the inviscid solution, it is clear that $R$ derivatives of physical quantities are generally $O(n)$. This will then certainly also be the case here. We write

$$
(P, F, G, H, \Gamma, \tau)=\left(\frac{1}{n} P_{0}, F_{0}, G_{0}, H_{0}, \Gamma_{0}, \tau_{0}\right)+\frac{1}{n}\left(\frac{1}{n} P_{1}, F_{1}, G_{1}, H_{1}, \Gamma_{1}, \tau_{1}\right)+\ldots
$$

The leading order terms in the momentum and continuity equations then give

$$
G_{0}=-\frac{\operatorname{sign}(n)}{2 q R} P_{0} \quad H_{0}=\frac{\operatorname{sign}(n)}{2 q n} P_{0 R}
$$

At the next order in $n$, the following results are obtained:

$$
\begin{aligned}
& \varphi_{F}+\varphi_{1} F_{0}=-P_{0}\left(\vec{\alpha}+\frac{1}{q}\right) \\
& 2 q H_{1}=\frac{\varphi_{1}}{2 q R} P_{0}-\Gamma_{0} q^{2} \operatorname{sign}(n) R+\frac{1}{n} \operatorname{sign}(n) P_{1 R}-\varphi_{G} \\
& 2 q G_{1}+\operatorname{sign}(n) \frac{P_{1}}{R}=-\frac{\varphi_{1}}{2 q n} \operatorname{sign}(n) P_{0 R}-\varphi_{H} \\
& \frac{H_{1}}{R}=-\frac{1}{n} G_{1 R}-\operatorname{sign}(n) \bar{\alpha} F_{0} \\
& \gamma \bar{M}^{2} P_{0}=\Gamma_{0}+\tau_{0}
\end{aligned}
$$




$$
(\gamma-1) \bar{M}^{2} \varphi_{1} P_{0}=\varphi_{1} \tau_{0}+\varphi_{r}
$$

Here

$$
\begin{gathered}
\varphi_{1}=\frac{1}{2} \varphi_{0}^{\prime \prime}(0) R^{2}-\bar{\alpha} c_{1} \\
\left(\begin{array}{c}
\varphi_{G} \\
\varphi_{H} \\
\varphi_{F} \\
\varphi_{\tau}
\end{array}\right)=\frac{i n^{5}}{R e}\left[\frac{1}{n^{2}} \frac{d^{2}}{d R^{2}}-\frac{1}{R^{2}}\right]\left(\begin{array}{c}
G_{0} \\
H_{0} \\
F_{0} \\
\tau_{0}
\end{array}\right) .
\end{gathered}
$$

We have implicitly assumed $R e=O\left(n^{5}\right)$; it is this scaling that leads to the key regime where viscosity substantially influences the stability of these flows. We therefore write $\mu_{1}=\frac{n^{5}}{R e}$. However the problem for $\mu_{1}=O(1)$ leads to an eighth-order system, almost of comparable computational difficulty to that of the full system as represented by (10). In order to make further progress without substantial computational effort, instead, we now assume the limit of diminishing viscosity, i.e. $\mu_{1} \rightarrow 0$, (but with $\left|\mu_{1}\right|>>\left|\frac{1}{n}\right|$ ). This enables us to write

$$
\begin{gathered}
\left(P_{0}, P_{1}, G_{0}, G_{1}, H_{0}, H_{1}, F_{0}, \tau_{0}, \Gamma_{0}, c_{1}\right)=\left(P_{00}, P_{10}, G_{00}, G_{10}, H_{00}, H_{10}, F_{00}, \tau_{00}, \Gamma_{00}, c_{10}\right) \\
+\mu_{1}\left(P_{01}, P_{11}, G_{01}, G_{11}, H_{01}, H_{11}, F_{01}, \tau_{01}, \Gamma_{01}, c_{11}\right)+\ldots
\end{gathered}
$$

It turns out, predictably, that $P_{00}$ is then the leading order inviscid solution, as found by Stott \& Duck (1994), and is described by

$$
\mathcal{L}\left\{P_{00}\right\}=P_{00 R R}+n^{2}\left[\frac{4 \bar{\alpha}^{2} q^{2}+4 \bar{\alpha} q}{\varphi_{10}^{2}}+\frac{2 q^{3} \bar{M}^{2}}{\varphi_{10}}-\frac{1}{R^{2}}\right] P_{00}=0
$$

After some algebra, the next order equation (in $\mu_{1}$ ) is given by

$$
\begin{aligned}
\mathcal{L}\left\{P_{01}\right\}= & -\frac{2 q n^{2} \operatorname{sign}(n) \tilde{\varphi}_{G}}{R \varphi_{10}}-\frac{2 q^{3} n^{2} \tilde{\varphi}_{\tau}}{\varphi_{10}^{2}}-\frac{2 q n \operatorname{sign}(n)\left(\tilde{\varphi}_{H}\right)_{R}}{\varphi_{10}}-\frac{4 q^{2} n^{2} \bar{\alpha} \tilde{\varphi}_{F}}{\varphi_{10}^{2}} \\
& -n^{2} P_{00}\left[2\left(4 q^{2} \bar{\alpha}^{2}+4 \bar{\alpha} q\right) / \varphi_{10}+2 q^{3} \bar{M}^{2}\right] \bar{\alpha} c_{11} / \varphi_{10}^{2}
\end{aligned}
$$

where

$$
\left(\begin{array}{c}
\tilde{\varphi}_{G} \\
\tilde{\varphi}_{H} \\
\tilde{\varphi}_{F} \\
\tilde{\varphi}_{\tau}
\end{array}\right)=\left(\begin{array}{c}
\frac{-i s i g n(n)}{2 q R}\left[\frac{1}{n^{2}} P_{00 R R}-\frac{P_{00}}{R^{2}}\right] \\
\frac{i s i g n(n)}{2 q n}\left[\frac{1}{n^{2}} P_{00 R R R}-\frac{P_{00 R}}{R^{2}}\right] \\
i\left[\frac{1}{n^{2}} F_{00 R R}-\frac{F_{00}}{R^{2}}\right] \\
\frac{i}{\sigma}\left[\frac{1}{n^{2}} \tau_{00 R R}-\frac{\tau_{00}}{R^{2}}\right]
\end{array}\right)
$$




$$
\begin{aligned}
& F_{00}=-\frac{1}{\varphi_{10}} P_{00}\left(\frac{1}{q}+\bar{\alpha}\right), \\
& \tau_{00}=(\gamma-1) \bar{M}^{2} P_{00}, \\
& \varphi_{10}=\frac{1}{2} \varphi_{0}^{\prime \prime}(0) R^{2}-\bar{\alpha} c_{10} .
\end{aligned}
$$

We then have, as $R \rightarrow 0$

$$
P_{00} \rightarrow R^{\frac{1}{2}}\left(A_{30} R^{|n|}+A_{40} R^{-|n|}\right), \quad P_{01} \rightarrow R^{\frac{1}{2}}\left(A_{31} R^{|n|}+A_{41} R^{-|n|}\right) .
$$

Since the solution must be bounded as $R \rightarrow 0$, we have $A_{40}=A_{41}=0$. In the limit as $R \rightarrow \infty$, we find

$$
P_{00} \rightarrow R^{\frac{1}{2}}\left(A_{10}^{*} R^{s}+A_{20}^{*} R^{-s}\right), \quad P_{01} \rightarrow R^{\frac{1}{2}}\left(A_{11}^{*} R^{s}+A_{21}^{*} R^{-s}\right),
$$

where $s=|n|\left(1-\frac{4 q^{3} \bar{M}^{2}}{\varphi_{0}^{\prime \prime}(0)}\right)^{\frac{1}{2}}$.

This solution must be matched to a layer described by $\bar{r}=r|n|^{\frac{1}{2}}=O(1)$, for which the following solution can be written

$$
P=\bar{r}^{\frac{1}{2}}\left(C_{1} I_{\nu}\left(\frac{q^{2} \bar{M}^{2}|n| \bar{r}^{2}}{4}\right)+C_{2} K_{\nu}\left(\frac{q^{2} \bar{M}^{2}|n| \bar{r}^{2}}{4}\right)\right) \exp \left(\frac{1}{2} \int|n|^{2} q^{2} \bar{r} \bar{M}^{2} d \bar{r}\right)
$$

where $I_{\nu}$ and $K_{\nu}$ are modified Bessel functions with $\nu=\frac{1}{2} s$. Note that $\nu$ (and also $s$ ) is imaginary. A relationship between $C_{1}$ and $C_{2}$ is determined from a consideration of the $r=O(1)$ region, which is inviscid in nature, and leads to a WKB-type solution, giving the result

$$
C_{1}=-2 i \pi C_{2} \exp \left\{\int_{r_{0}}^{0}|n|^{2}\left(\left[-\bar{M}^{2} \varphi_{0}^{2}+\frac{W^{4} \bar{M}^{4}}{4 r^{2}}\right]^{\frac{1}{2}}\right) d r\right\}^{2}
$$

where $r_{0}$ is determined from

$$
\varphi_{0}^{2}\left(r_{0}\right)=\frac{W^{4}\left(r_{0}\right) \bar{M}^{2}}{4 r_{0}^{2}}
$$

Matching asymptotically, and taking the limit $|n| \rightarrow \infty$, leads to the conclusion (on account of $(43))$

$$
A_{10}^{*}=A_{11}^{*}=\ldots=0 .
$$


Equation (34) may be solved using hypergeometric functions (Stott \& Duck 1994), and this leads to

$$
c_{10}=-\frac{2 n^{2} q(1+q \bar{\alpha})}{\left[\phi_{0}^{\prime \prime}(0)\right] \tilde{\mu}(\tilde{\mu}+1)},
$$

where $\tilde{\mu}=\frac{1}{2}[|n|+s]+N, N=0,1,2,3 \ldots$ Alternatively a WKB solution would also appear to be in order here, although this is not straightforward due to the multitude of critical points. Since the system (35) depends on a largely numerical approach, we chose to adopt a numerical approach for the problem overall, including (34), treating $n$ as a finite (but large) numerical parameter, using a fourth order Runge-Kutta scheme.

This analysis differs somewhat from that of Stewartson \& Brown (1985), who looked at near neutral centre modes. In their work $s$ was real (and so too, consequently was $c_{10}$ ), and it was necessary to seek higher order terms in order to obtain the imaginary part of $c_{1}$. In the following section, we consider some numerical results arising from the analysis of this and the previous section.

\section{Conclusions}

In the work described in the previous two sections we find that as in the corresponding inviscid analysis, the Mach number proves to be crucially dependent on the order of the wavenumber and we are able to develop the analysis in a similar way to the previous inviscid study. Note that for all results referred to in this section the swirl parameter $q$ is taken to be 0.8 .

Khorrami (1992) noted that modes evaluated above Reynolds number as low as 10000 , attain their maximum growth rates and beyond this point appear to be largely independent of the Reynolds number. Figure 1 shows a plot of growth rate against Reynolds number for $n=-15$ with $\alpha=7.5$ with $M=|n|^{\frac{1}{2}}$ (for the first mode i.e. that corresponding to $m=0$ in (24)). We observe from these results that stabilisation occurs for sufficiently low Reynolds number; this is a generic feature of this problem (and indeed also occurs in the incompressible case, generally).

Figure 2 shows the neutral curve ( $\alpha$ against Reynolds number) for the case $n=-15$ with $M=\mathrm{O}\left(|n|^{\frac{1}{2}}\right)$ (again for the first mode), and confirms the remarks regarding the effects of stabilisation above.

Figure 3 shows the variation of the growth rates with Mach number for the most unstable mode for varying Reynolds numbers and $n=-15$, with $M=\mathrm{O}\left(|n|^{\frac{1}{2}}\right)$. In this figure it can be seen that for all Reynolds numbers considered the effect of increasing Mach number is generally stabilising and for sufficiently large $M$ modes are completely stabilised. Also as the Reynolds number is increased the value of the Mach number at which the modes become stable decreases.

We now turn to consider the effects of viscosity on centre modes. Figures $4 \mathrm{a}-4 \mathrm{c}$ show growth rate variations for the case $M=15, n=-15, q=0.8, \sigma=1$, for $R e=\infty$ (fig. 4a), $R e=2.5 \times 10^{6}$ (fig. 4b) and $R e=10^{6}$ (fig. 4c). These figures show the first three modes, with the first mode $(N=0)$ shown as a solidus, the second mode $(N=1)$ shown as the larger dashed line, and the third mode $(N=2)$ shown as the smaller dashed line. 
The first of these figures, namely fig. 4a corresponds with the inviscid limit, as considered by Stott \& Duck (1994), and agrees very favourably with these previous results, obtained by (46). These results also show the trend observed by Stott \& Duck (1994), namely that near the lower neutral point ( $\alpha \rightarrow 2$ in this case), that the higher modes do have slightly larger growth rates than corresponding lower modes. Figures $4 \mathrm{~b}, 4 \mathrm{c}$ do generally indicate that viscosity plays a stabilising effect on all three modes considered. Indeed, these observed trends are, again, quite generic, and are observed in general calculations of this problem.

\section{Acknowledgements}

This research was supported by the National Aeronautics and Space Administration under NASA Contract No. NASA-18605 while one of the authors was in residence at the Institute for Computer Applications in Science and Engineering (ICASE), NASA Langley Research Center, Hampton, VA. J.A.K.S was in receipt of a S.E.R.C. studentship. A number of the computations were performed with the computer facilities provided by the University of Manchester, and by S.E.R.C. under grants GR/H 83683 and GR/H 58568.

\section{References}

Batchelor, G.K. 1964 Axial flow in trailing line vortices. J. Fluid Mech. $\underline{20}, 645$

Duck, P.W. 1986 The inviscid stability of a swirling flow: large wavenumber disturbance. J. Appl. Math. Phys. (ZAMP) $\underline{37}, 340$

Duck, P.W. \& Khorrami, M.R. 1992 On the effects of viscosity on the stability of a trailing line vortex. J. Fluid Mech. 245, 175 (Also ICASE report 91-6)

Khorrami, M.R. 1991 On the viscous modes of instability of a trailing line vortex. J. Fluid Mech. 225, 197

Khorrami, M.R. 1992 Behaviour of asymmetric unstable modes of a trailing-line vortex near the upper neutral curve. Phys. Fluids A $\underline{4}, 1310$.

Leibovich, S. \& Stewartson, K. 1983 A sufficient condition for the instability of columnar vortices. J. Fluid Mech. $\underline{126}, 355$

Lessen, M. \& Paillet, F. 1974 The stability of a trailing line vortex. Part 2. Viscous theory. J. Fluid Mech. $\underline{65}, 769$

Lessen, M., Singh, P. \& Paillet, F. 1974 The stability of a trailing line vortex. Part 1. Inviscid theory. J. Fluid Mech. $\underline{53}, 753$

Stewartson, K. 1982 The stability of swirling flows at large Reynolds number when subjected to disturbances with large azimuthal wavenumber. Phys. Fluids $\underline{25}, 11$

Stewartson, K. \& Brown, S.N. 1985 Near neutral centre modes as inviscid perturbations to a trailing line vortex. J. Fluid Mech. $\underline{156}, 387$

Stott, J.A.K. \& Duck, P.W. 1994 The stability of a trailing-line vortex in compressible flow. J. Fluid Mech $\underline{268}, 323$ (also ICASE Report No. 92-65) 
Figure 1: Variation of growth rates against Reynolds number for $M=|n|^{\frac{1}{2}}, n=-15$, $\alpha=7.5$.

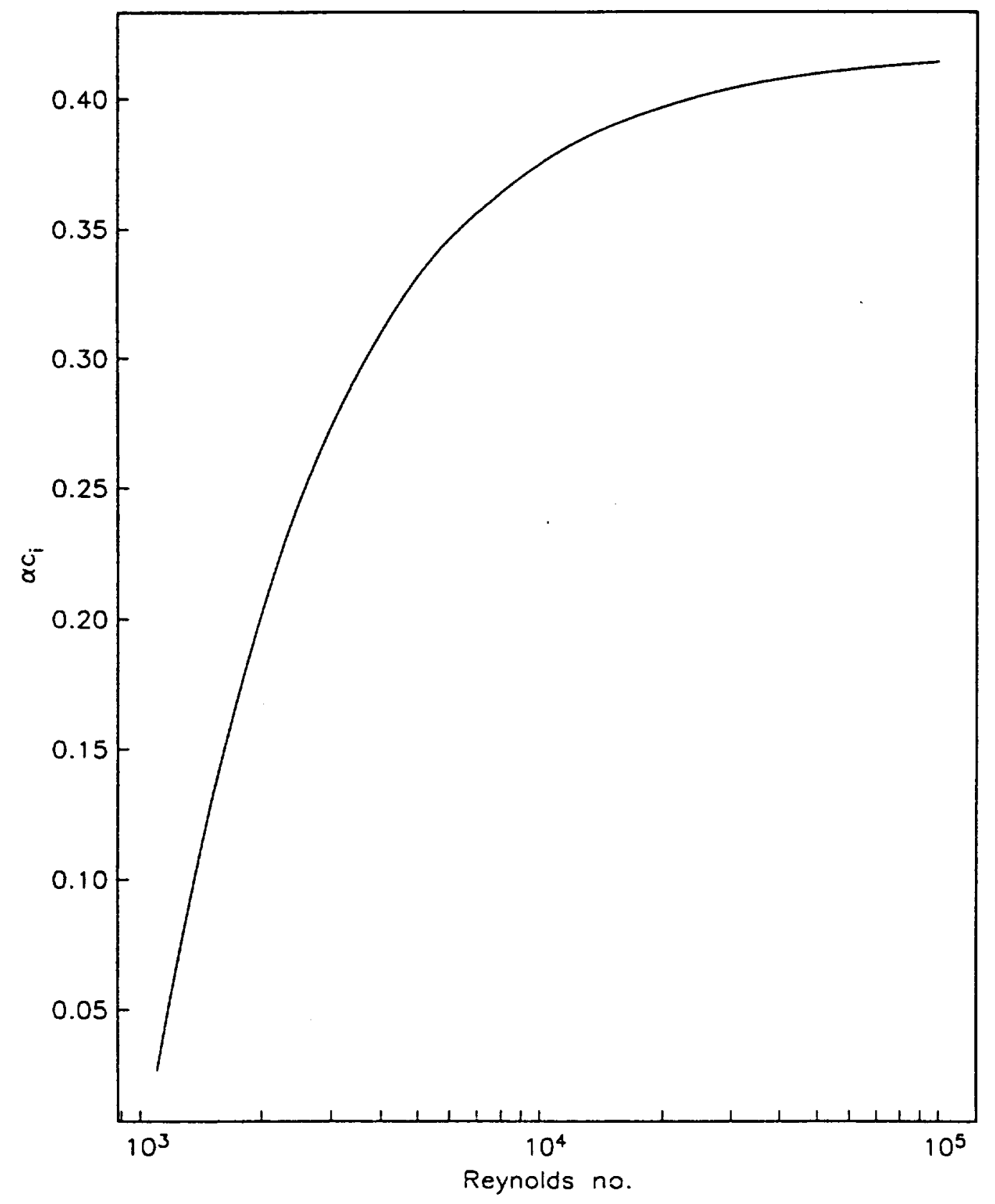


Figure 2: Neutral Curves for $M=|n|^{\frac{1}{2}}, n=-15$.

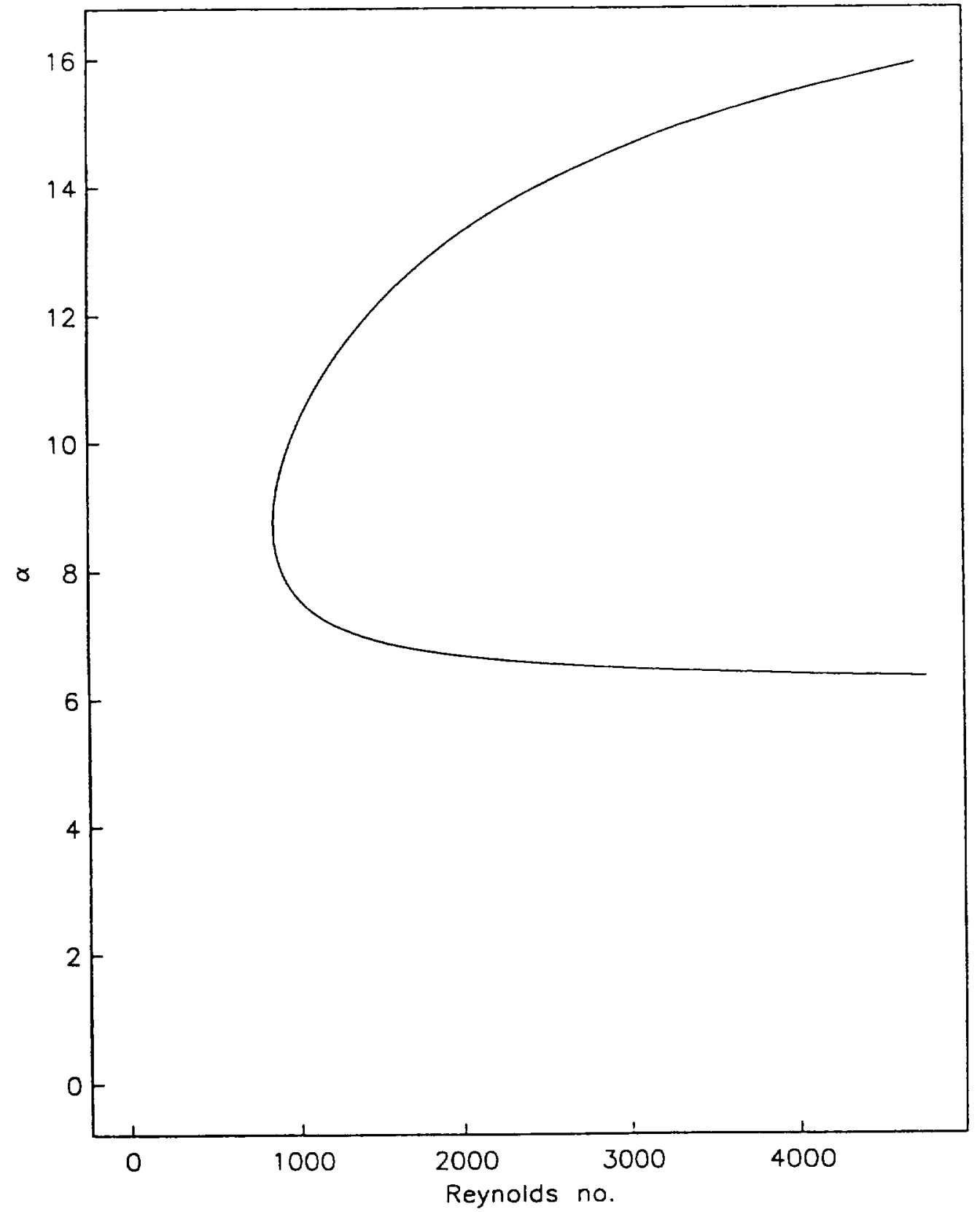


Figure 3: Variation of growth rate with Mach number for $n=-15, \alpha=7.5$ with $R e=$ $100000(-), R e=10000(---)$, and $R e=1500(--)$.

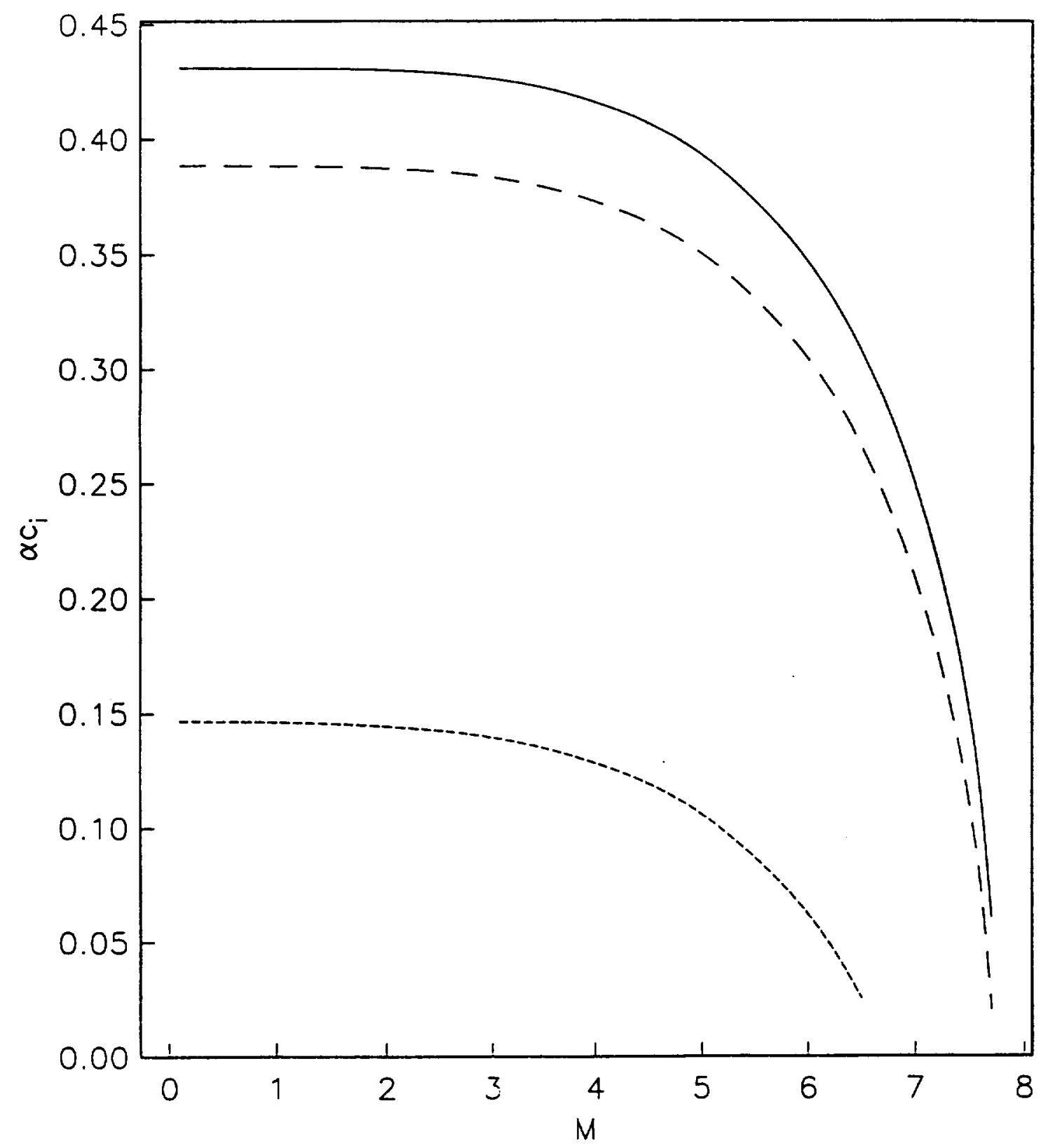


Figure 4a: Variation of growth rate with $\alpha$ for centre modes $n=-15, M=15, q=0.8$ $R e=\infty$.

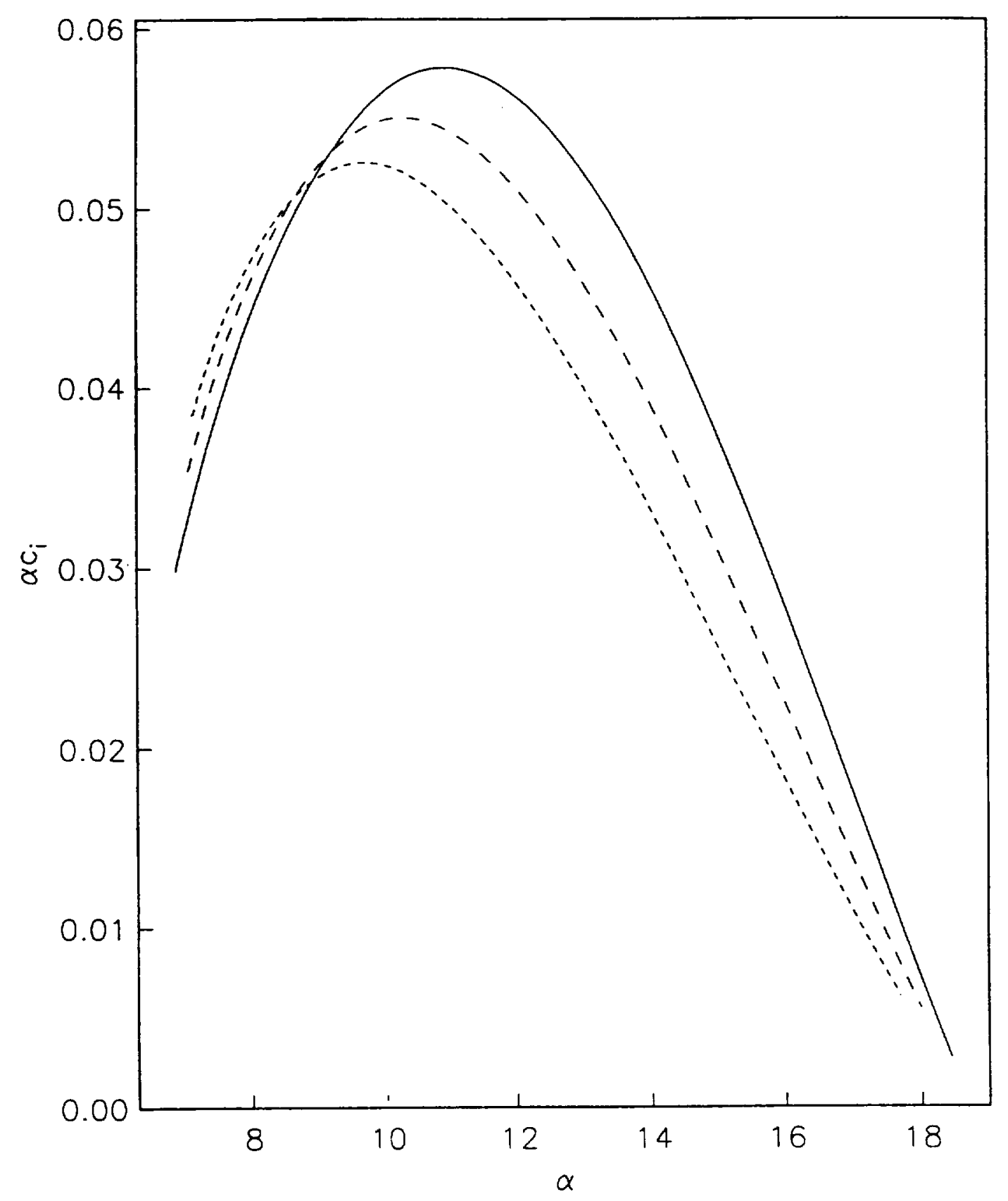


Figure 4b: Variation of growth rate with $\alpha$ for centre modes $n=-15, M=15, q=0.8$ $R e=2.5 \times 10^{6}$.

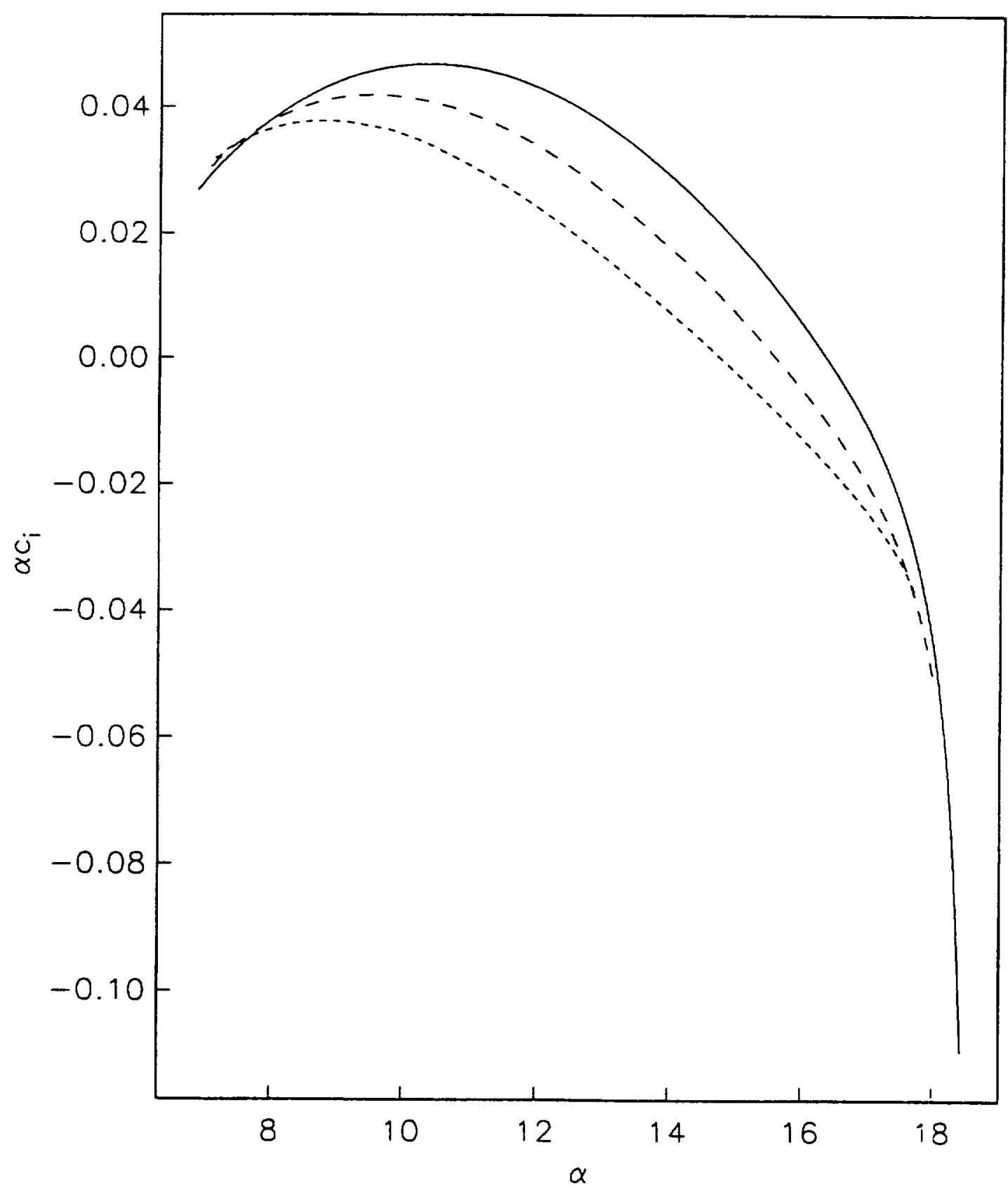


Figure 4c: Variation of growth rate with $\alpha$ for centre modes $n=-15, M=15, q=0.8$ $R e=10^{6}$.

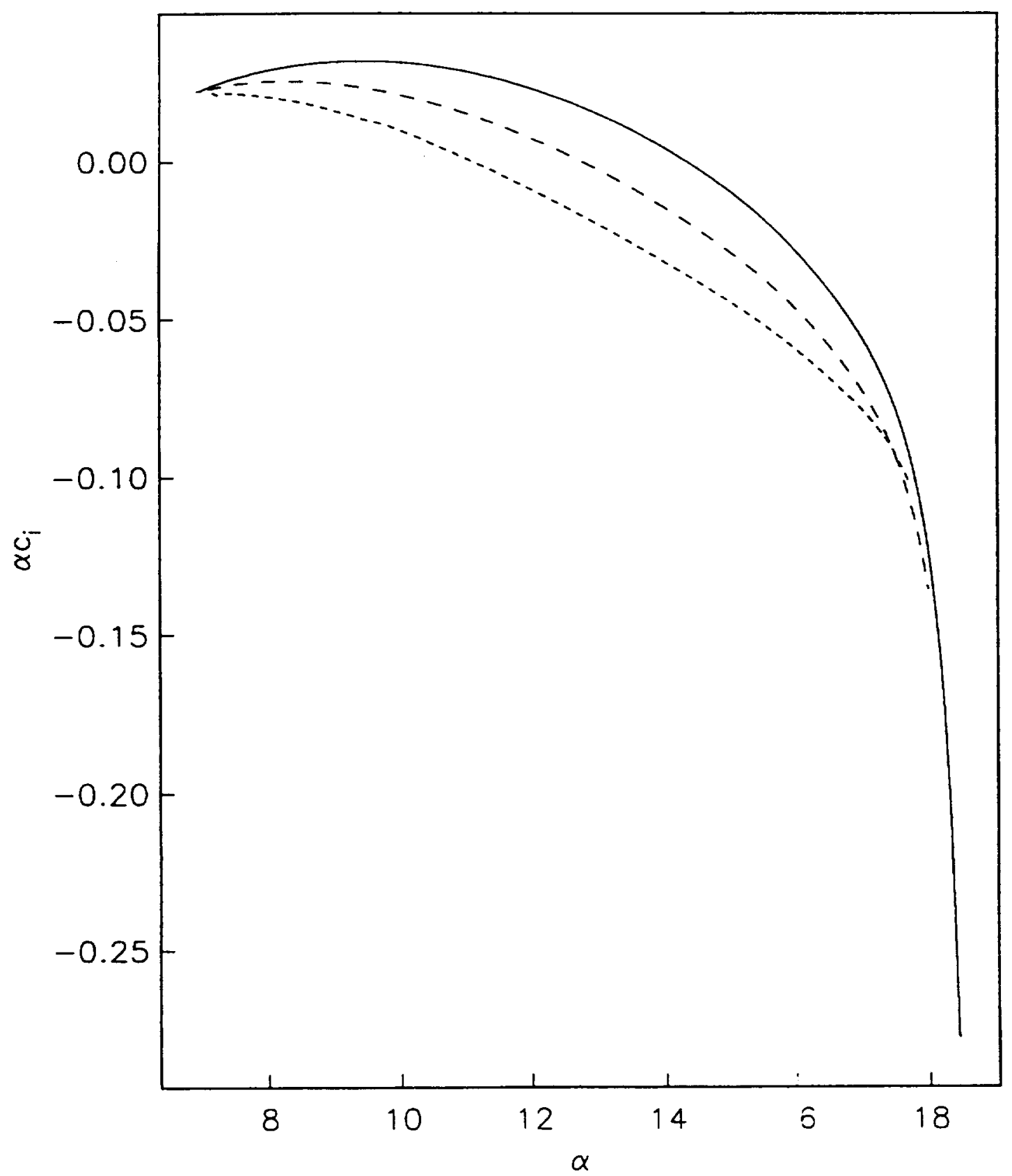






\begin{tabular}{|c|c|c|}
\hline \multicolumn{2}{|c|}{ REPORT DOCUMENTATION PAGE } & $\begin{array}{l}\text { Form Approved } \\
\text { OMB No. 0704-0188 }\end{array}$ \\
\hline \multicolumn{3}{|c|}{ 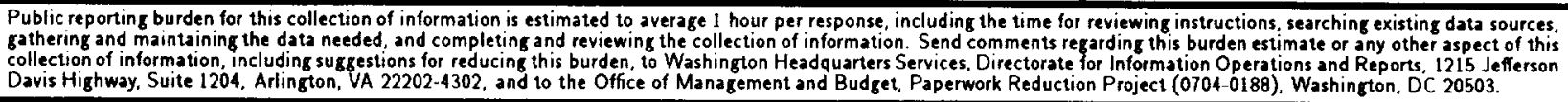 } \\
\hline \multicolumn{2}{|c|}{\begin{tabular}{l|l|l|} 
1. AGENCY USE ONLY(Leave blank) & $\begin{array}{l}\text { 2. REPORT DATE } \\
\text { July } 1994\end{array}$ & 3. \\
4. TITLE AND SUBTITLE &
\end{tabular}} & $\begin{array}{l}\text { VD DATES COVERED } \\
\text { Ort }\end{array}$ \\
\hline \multicolumn{2}{|c|}{$\begin{array}{l}\text { 4. TITLE AND SUBTITLE } \\
\text { THE EFFECTS OF VISCOSITY ON THE STABILITY OF A } \\
\text { TRAILING-LINE VORTEX IN COMPRESSIBLE FLOW }\end{array}$} & $\begin{array}{l}\text { 5. FUNDING NUMBERS } \\
\text { C NAS1-19480 } \\
\text { WU } 505-90-52-01\end{array}$ \\
\hline \multicolumn{2}{|l|}{$\begin{array}{l}\text { 6. AUTHOR(S) } \\
\text { Jillian A. K. Stott } \\
\text { Peter W. Duck }\end{array}$} & \\
\hline \multicolumn{2}{|c|}{$\begin{array}{l}\text { 7. PERFORMING ORGANIZATION NAME(S) AND ADDRESS(ES) } \\
\text { Institute for Computer Applications in Science } \\
\text { and Engineering } \\
\text { Mail Stop 132C, NASA Langley Research Center } \\
\text { Hampton, VA 23681-0001 }\end{array}$} & $\begin{array}{l}\text { 8. PERFORMING ORGANIZATION } \\
\text { REPORT NUMBER } \\
\text { ICASE Report No. } 94-58\end{array}$ \\
\hline \multicolumn{2}{|c|}{$\begin{array}{l}\text { 9. SPONSORING/MONITORING AGENCY NAME(S) AND ADDRESS(ES) } \\
\text { National Aeronautics and Space Administration } \\
\text { Langley Research Center } \\
\text { Hampton, VA } 23681-0001\end{array}$} & $\begin{array}{l}\text { 10. SPONSORING/MONITORING } \\
\text { AGENCY REPORT NUMBER } \\
\text { NASA CR-194948 } \\
\text { ICASE Report No. } 94-58\end{array}$ \\
\hline \multirow{2}{*}{\multicolumn{3}{|c|}{$\begin{array}{l}\text { 11. SUPPLEMENTARY NOTES } \\
\text { Langley Technical Monitor: Michael F. Card } \\
\text { Final Report } \\
\text { Submitted to Physics of Fluids }\end{array}$}} \\
\hline & & \\
\hline \multirow{2}{*}{\multicolumn{2}{|c|}{$\begin{array}{l}\text { 12a. DISTRIBUTION/AVAILABILITY STATEMENT } \\
\text { Unclassified-Unlimited } \\
\text { Subject Category } 34\end{array}$}} & 126. OISTRIBUTION CODE \\
\hline & & \\
\hline \multicolumn{3}{|c|}{$\begin{array}{l}\text { 13. ABSTRACT (Maximum } 200 \text { words) } \\
\text { We consider the effects of viscosity on the inviscid stability of the Batchelor (1964) vortex in a compressible flow. The } \\
\text { problem is tackled asymptotically, in the limit of large (streamwise and azimuthal) wavenumbers, together with large } \\
\text { Mach numbers. This problem, with viscous effects neglected, was discussed in Stott \& Duck (1994). The authors } \\
\text { found that the nature of the solution passes through different regimes as the Mach number increases, relative to the } \\
\text { wavenumber. This structure persists when viscous effects are included in the analysis. In the present study, as in } \\
\text { that mentioned above, the mode present in the incompressible case ceases to be unstable at high Mach numbers and } \\
\text { a centre mode forms, whose stability characteristics are determined primarily by conditions close to the vortex axis. } \\
\text { We find generally that viscosity has a stabilising influence on the flow, whilst in the case of centre modes, viscous } \\
\text { effects become important at much larger Reynolds numbers than for the first class of disturbance. }\end{array}$} \\
\hline
\end{tabular}

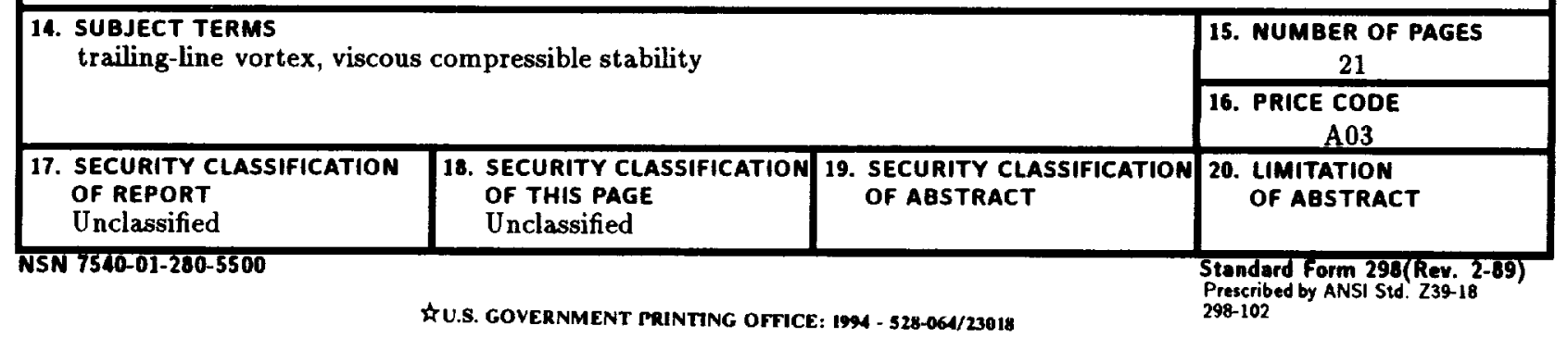

